\title{
Chebyshev Descriptors for SHM with Acoustic Emission and Acousto Ultrasonics
}

\author{
D. Crivelli ${ }^{*}$, M. Eaton ${ }^{2}$, M. Pearson ${ }^{2}$, K. Holford ${ }^{2}$, R. Pullin ${ }^{2}$ \\ ${ }^{1}$ Department of Mechanical Engineering, Politecnico di Milano, Milano, Italy \\ ${ }^{2}$ Cardiff School of Engineering, Cardiff University, Cardiff, UK
}

\section{Structured Abstract:}

Purpose - The paper is a feasibility study on the use of alternative parameters for representing Acoustic Emission and Acousto Ultrasonic signals, using a wavelet based approach and the computation of Chebyshev moments.

Design/methodology/approach - Two tests were performed, one on Acoustic Emission artificial signals generated on a CFRP plate and one on an Acousto Ultrasonic setup used for actively detecting impact damage. The waveforms were represented using a data reduction technique based on the Daubechies wavelet and an image processing technique using Chebyshev moments approximation, to get 32 descriptors for each waveform.

Findings - The use of such descriptors allowed in the Acoustic Emission case to verify that the moments are similar when the waveforms are similar; in the Acousto Ultrasonic setup the correlation coefficient of the descriptors with respect to a reference dataset was found to be linked to the delimitation size.

Practical implications - Such a data reduction while retaining all the useful information will be positive for wireless sensor networks, where power consumption during data transmission is key. With having to send only a reliable set of descriptors and not an entire waveform, the power consumption is believed to be reduced.

Originality/value - This paper is a preliminary study that fulfills a need for a more reliable data reduction for ultrasonic transient signals, such as those used in Acoustic Emission and Acousto Ultrasonics.

Keywords: Acoustic Emission, Acousto-Ultrasonics, Structural Health Monitoring, Damage Detection, Wavelets, Chebyshev moments

Article Classification: Research Paper

\section{Introduction}

Many techniques based on passive and active ultrasonic waves are being increasingly studied for their use as a structural health monitoring (SHM) system for large structures, such as bridges or aircrafts, where the need to monitor the structure's status in real time is vital (Kapoor et al. 2009). 
Among these techniques, Acoustic Emission (AE) and Acousto-Ultrasonics (AU) are the most attractive at the moment. The former is based on the passive recording of ultrasonic transient waveforms emitted by materials undergoing damage (Finleyson 2003) by means of piezoelectric sensors; it is well established that different damage types or sources cause different waveforms to be emitted by the material. The latter is based on the comparison between ultrasonic signals emitted by a source (actuator) and the same signal recorded at a certain distance (sensor); the difference between the recorded signal on an undamaged material and on a damaged material reflects itself in a shape change in the recorded waveform (Schulz et al. 2000).

It is clear that both techniques require a certain degree of advanced signal processing, especially for comparing different waveforms, but also require a certain level of data reduction. In fact, having to deal with the ultrasonic range, it is not uncommon to reach sampling rates of around $10 \mathrm{MHz}$, which results in a very large amount of data that has to be transmitted from the sensor to the data processing unit. For this purpose, AE traditionally uses parameters that are based on the waveform shape and characteristics (and are easy to compute in reasonably cheap programmable microprocessors), such as peak amplitude, duration, number of signal threshold-crossings, and others (Stone \& Dingwall 1977). As some authors (Eaton 2009) have pointed out though, these parameters are far from being ideal: they are not fully independent (as some are distinctly related to others) and heavily depend on the acquisition system setup. There is some effort being made in this direction to employ additional frequency based parameters, which has led to some results when supported by machine learning algorithms (Crivelli et al. 2014) or more traditional feature-space based reduction methods, as Principal Component Analysis (PCA) (Eaton et al. 2011).

Wavelet decomposition, or Discrete Wavelet Transform (DWT) is useful to provide information on timefrequency content of transient waveforms (Downs et al. 2003) and to separate noise and signal contributions; in the AE field many have used different types of wavelet transform mainly to identify different propagation modes and their behaviour. However, to the knowledge of the authors, no application so far has used their full potential to identify different waveforms in an efficient way (e. g. not relying on visual or manual sorting).

The field of image processing provides interesting examples of techniques to assess the similarity between two images. In particular, the use of Chebyshev [1] polynomials decomposition moments has been successfully used in image comparison, compression and detection (Mukundan et al. 2001); it has also been used in autofocus algorithms (Yap \& Raveendran 2004). In the mechanical engineering field, the computation of Chebyshev moments has been used by (Sebastian et al. 2011) to successfully and quantitatively compare results from two different deformation fields retrieved from Digital Image Correlation (DIC) on a real world experiment, and a deformation field from a Finite Element analysis (FEA) of the same test.

In this paper, a novel way to describe waveforms is suggested, which is meant to improve the existing classification algorithms and to provide a reasonable data reduction for transient wave analysis. The 
technique is evaluated against two datasets, the first involving AE calibration signals, and the second using Acousto-Ultrasonic data used to detect and quantify impact damage.

\section{Materials and methods}

\subsection{Chebyshev Descriptors extraction}

The application proposed for transient waveforms analysis can be summarized as follows:

1. Capture a discrete time waveform sampled at $\mathbf{d}_{\mathbf{i}}$ points (Figure 1a);

2. Compute a wavelet transform of the original waveform with an appropriate number of detail levels. In this case the Daubechies10 (Daubechies 1988) wavelet is used, up to detail 11 (Figure $1 b)$;

3. Rectify the signal or the wavelet transform;

4. Remove the unwanted levels which may represent low and high frequency noise;

5. Create a $\mathbf{M} \times \mathbf{N}$ matrix of $\mathbf{w}_{\mathbf{i}, \mathbf{n}}$ points (where $\mathbf{i}$ is the wavelet sample, and $\mathbf{n}$ is the wavelet detail level) (Figure 1c);

6. The Chebyshev moments of the $\mathbf{M} \times \mathbf{N}$ matrix are computed as described in (Bateman et al. 1955), up to an appropriate level $\mathbf{D}$ (in this case 36 is used, but the actual minimum required level might be lower) Table 1.

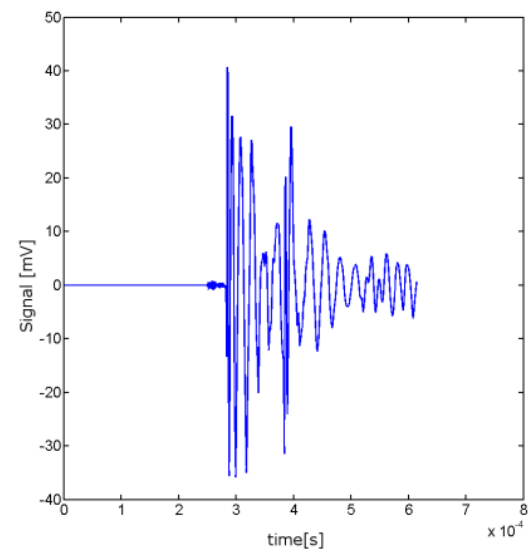

(a)

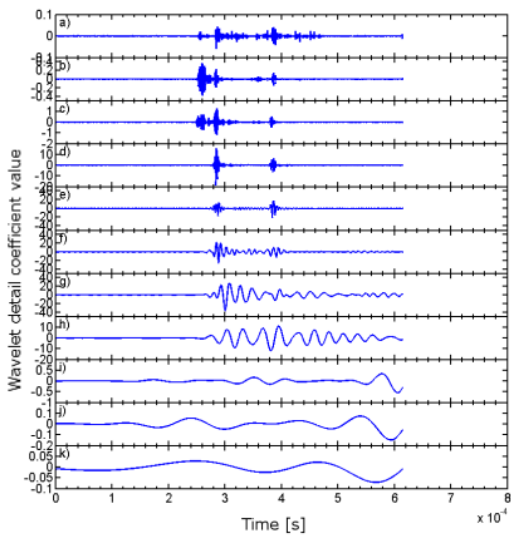

(b)

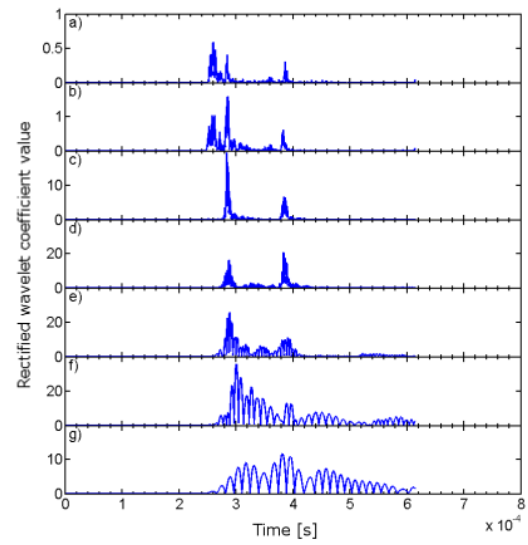

(c)

Figure 1: signal processing from the waveform to the rectified wavelet transform

At this point, a set of $\mathbf{D}$ descriptors that describe the waveform is obtained. By computing the Chebychev Descriptors (CDs) for two similar waveforms and plotting them in a scatter plot (using the $\mathrm{X}$ axis for the first waveform and the $\mathrm{Y}$ axis for the second waveform CDs), an immediate picture of the waveform similarity is highlighted: as the points get closer to the $\mathrm{x}=\mathrm{y}$ line, the highest degree of similarity between the two waveforms is found. 
Table 1: Chebyshev moments values

\begin{tabular}{|c|c|c|c|c|c|}
\hline $1.09 \mathrm{E}+00$ & $7.64 \mathrm{E}-01$ & $-1.63 \mathrm{E}+00$ & $-2.58 \mathrm{E}-06$ & $3.31 \mathrm{E}-06$ & $-4.04 \mathrm{E}-06$ \\
\hline $1.70 \mathrm{E}+00$ & $1.42 \mathrm{E}+00$ & $-2.21 \mathrm{E}+00$ & $3.58 \mathrm{E}-07$ & $-4.59 \mathrm{E}-07$ & $5.61 \mathrm{E}-07$ \\
\hline $2.87 \mathrm{E}-01$ & $6.77 \mathrm{E}-01$ & $2.48 \mathrm{E}-01$ & $4.45 \mathrm{E}-07$ & $-5.72 \mathrm{E}-07$ & $6.99 \mathrm{E}-07$ \\
\hline$-5.54 \mathrm{E}-03$ & $6.44 \mathrm{E}-04$ & $1.07 \mathrm{E}-02$ & $6.69 \mathrm{E}-07$ & $-8.59 \mathrm{E}-07$ & $1.05 \mathrm{E}-06$ \\
\hline $3.32 \mathrm{E}-03$ & $-3.86 \mathrm{E}-04$ & $-6.42 \mathrm{E}-03$ & $-4.01 \mathrm{E}-07$ & $5.15 \mathrm{E}-07$ & $-6.30 \mathrm{E}-07$ \\
\hline$-1.29 \mathrm{E}-03$ & $1.50 \mathrm{E}-04$ & $2.50 \mathrm{E}-03$ & $1.56 \mathrm{E}-07$ & $-2.00 \mathrm{E}-07$ & $2.45 \mathrm{E}-07$ \\
\hline
\end{tabular}

\subsection{Propagation study dataset}

A preliminary dataset, extracted from pencil-lead break signals (HSU 1976) recorded with Acoustic

Emission sensors on a carbon fiber panel at different locations and angles from the sensor was used to assess the general correctness of this approach.

The sensor used in this setup was a conical broadband sensor, held down with a weight and connected to a Physical Acoustics Corporation (PAC) pre-amplifier, connected to a Physical Acoustic Limited (PAL) PCI2 acquisition unit. Waveforms were sampled at $10 \mathrm{MHz}$ sampling rate. The sensor was placed in the centre of a $1 \mathrm{~m} \times 1 \mathrm{~m}$ carbon fibre laminate panel (Figure 2). Signals were generated with the pencil-lead break method (HSU 1976) at various positions on the panel.

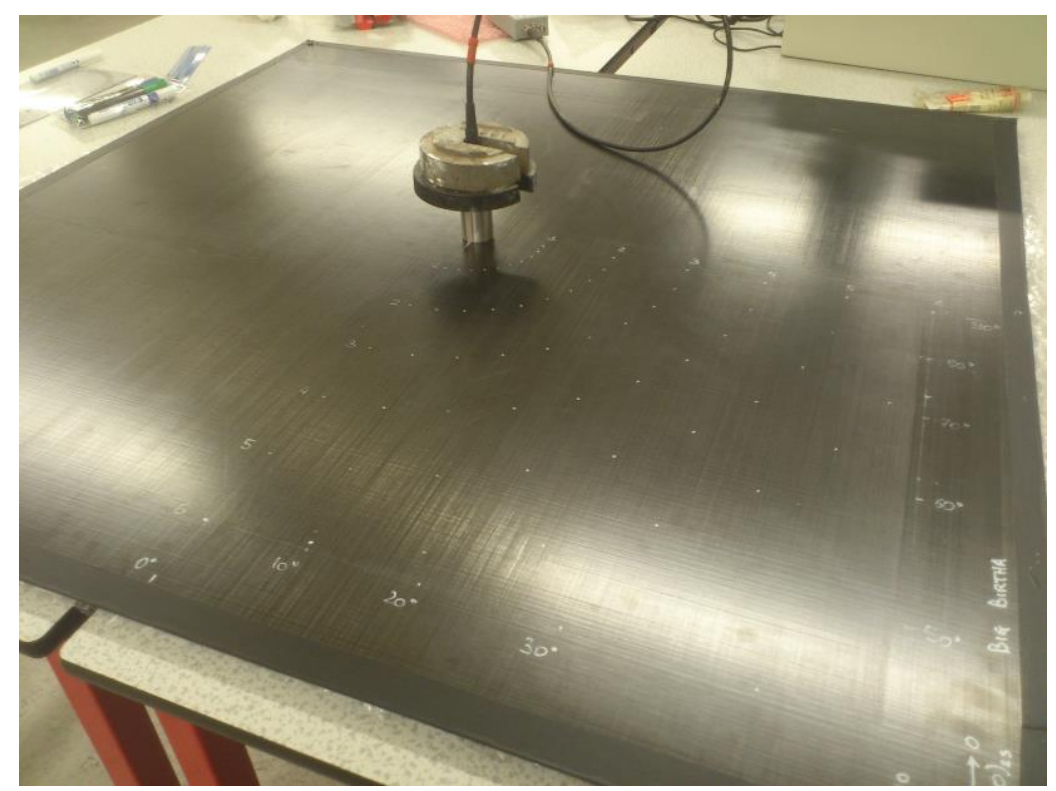

Figure 2: panel and sensor setup 


\subsection{Acousto-ultrasonic dataset}

The original experiment was published in (Pearson et al. 2011); briefly, it consists of acarbon fibre panel, $500 \mathrm{~mm} \times 500 \mathrm{~mm}$, from 8 plies of woven CFRP. Two M2807-P2 MFC transducers were attached to the panel using cyanoacrycate; this configuration can be seen in Figure 3.

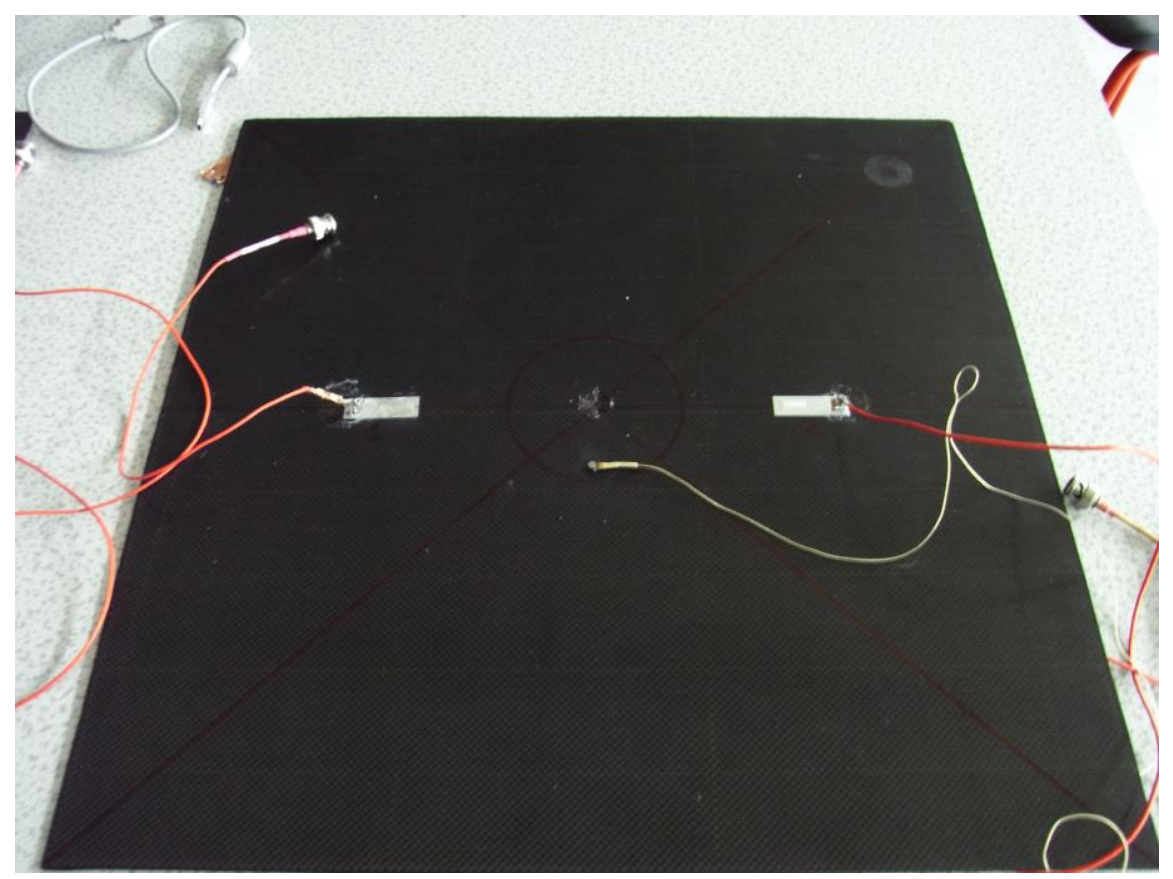

Figure 3: acousto-ultrasonics panel set-up

The panel was clamped in an impact testing machine and a set of $34 \mathrm{~J}$ impacts were performed between the two sensors. Before the first impact and after each subsequent impact one of the transducers was used to pulse a $100 \mathrm{kHz}$ square wave, while the other recorded the waveform at distance. Figure 4 shows the crosscorrelation between the baseline received signals and the impact signals; the delamination area was quantified using a C-scanner.

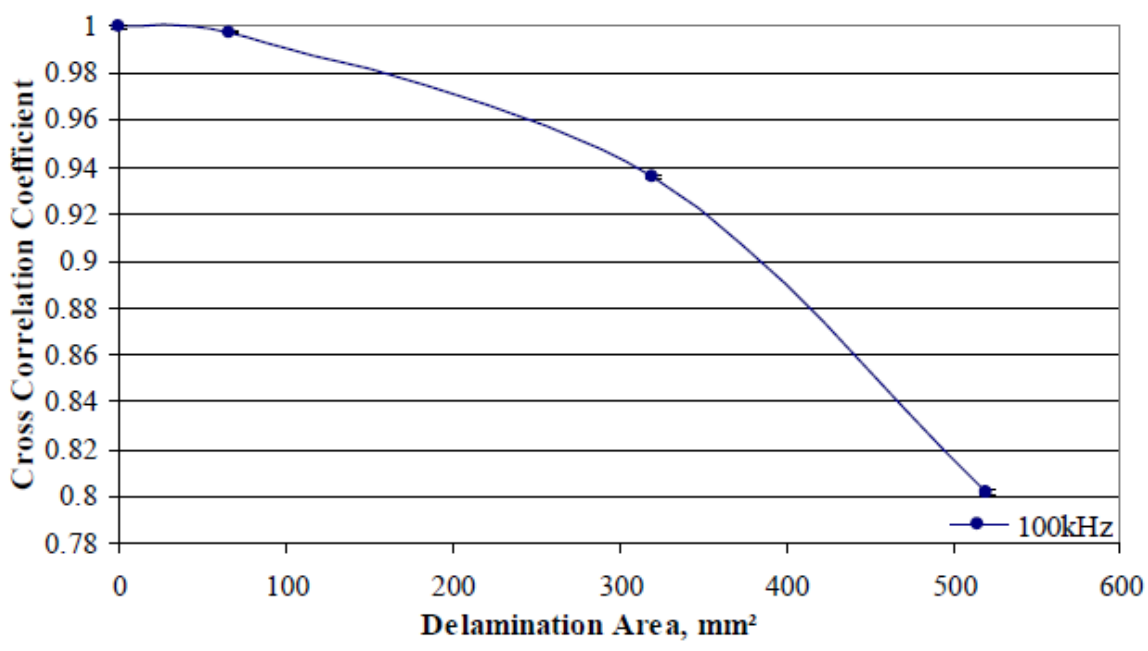

Figure 4: comparison between delamination area and waveform cross-correlation coefficient (Pearson et al. 2011) 


\section{Results}

\subsection{Pencil lead breaks}

Three waveforms, two from good pencil-lead breaks (plb1 and plb2) and one from a bad pencil lead break (double break) were acquired (Figure 5). It was found that the $\mathrm{R}^{2}$ measure of the goodness of fit of the CDs is an appropriate measure of the similarity between the two waveforms.

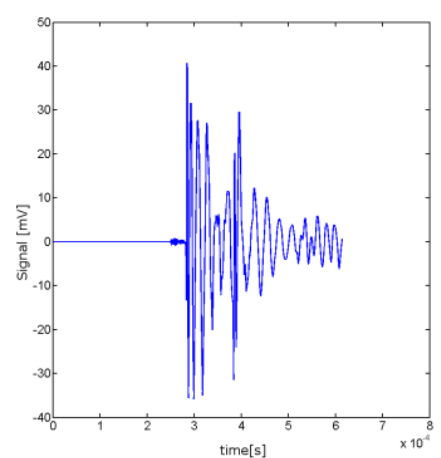

(a) plb1

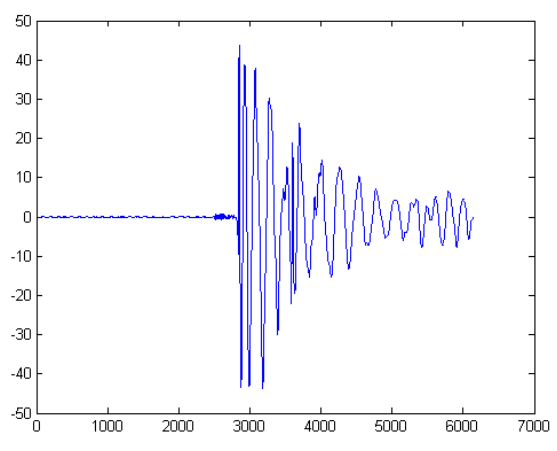

(b) plb2

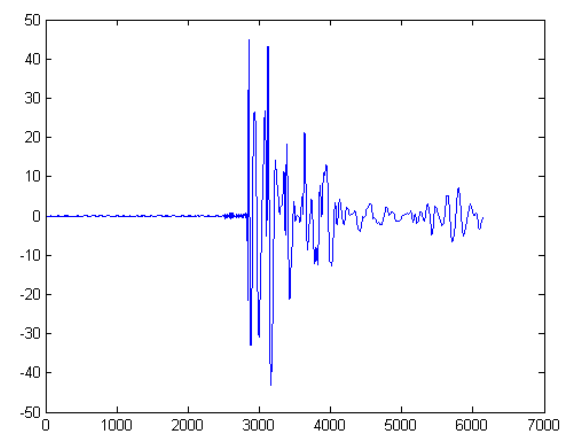

(c) bad break

Figure 5: pencil lead break waveforms used for the Acoustic Emission dataset

The comparisons between the signals are shown in Figure 6. The correlation coefficients for each pair comparison were computed; their values are reported in Table 2.

Table 2 : correlation coefficients for the 3 pairs comparisons

\begin{tabular}{|l|l|l|l|}
\hline & Plb1 vs plb2 & Plb1 vs bad break & Plb2 vs bad break \\
\hline Corr. coef & 0.9605 & 0.8550 & 0.8225 \\
\hline
\end{tabular}




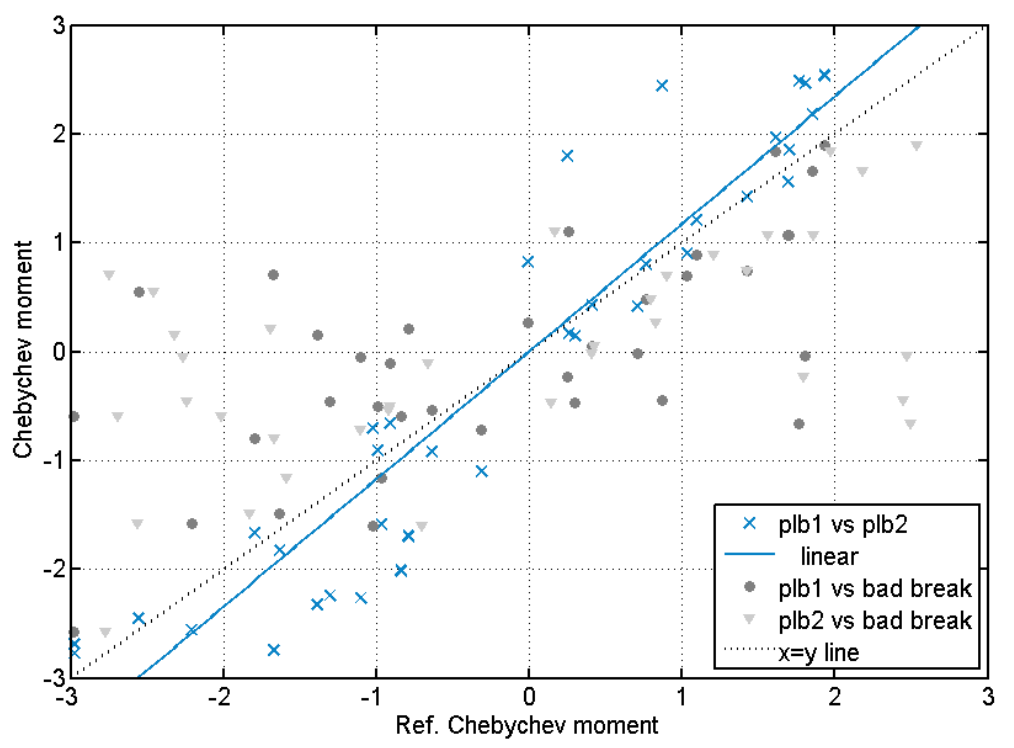

(a)

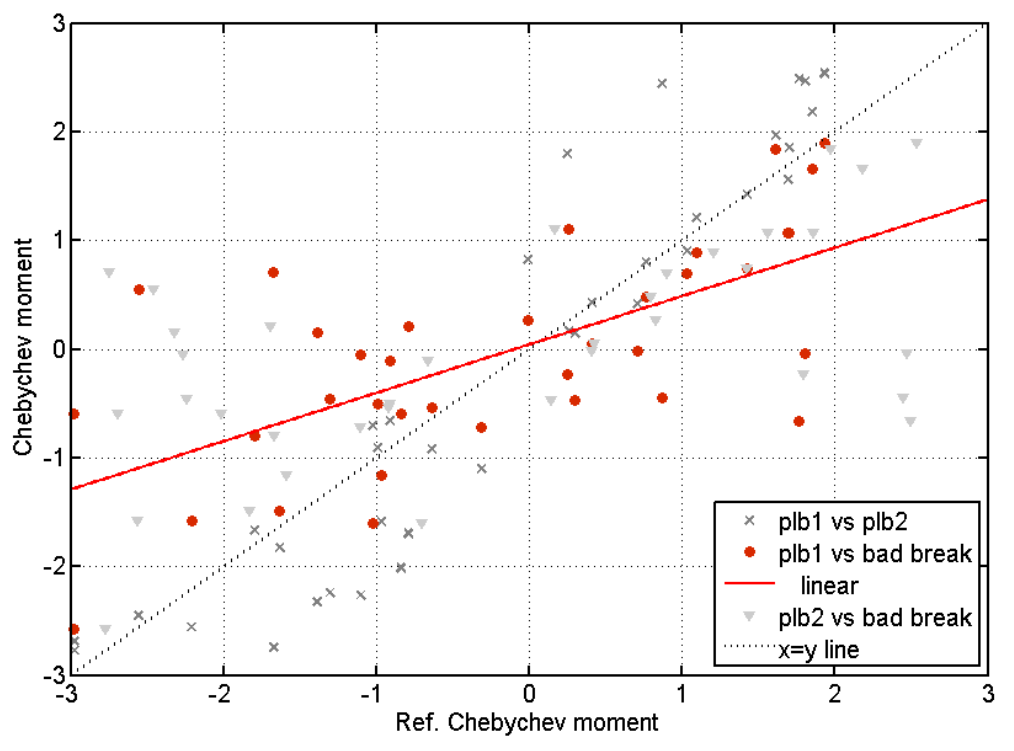

(b) 


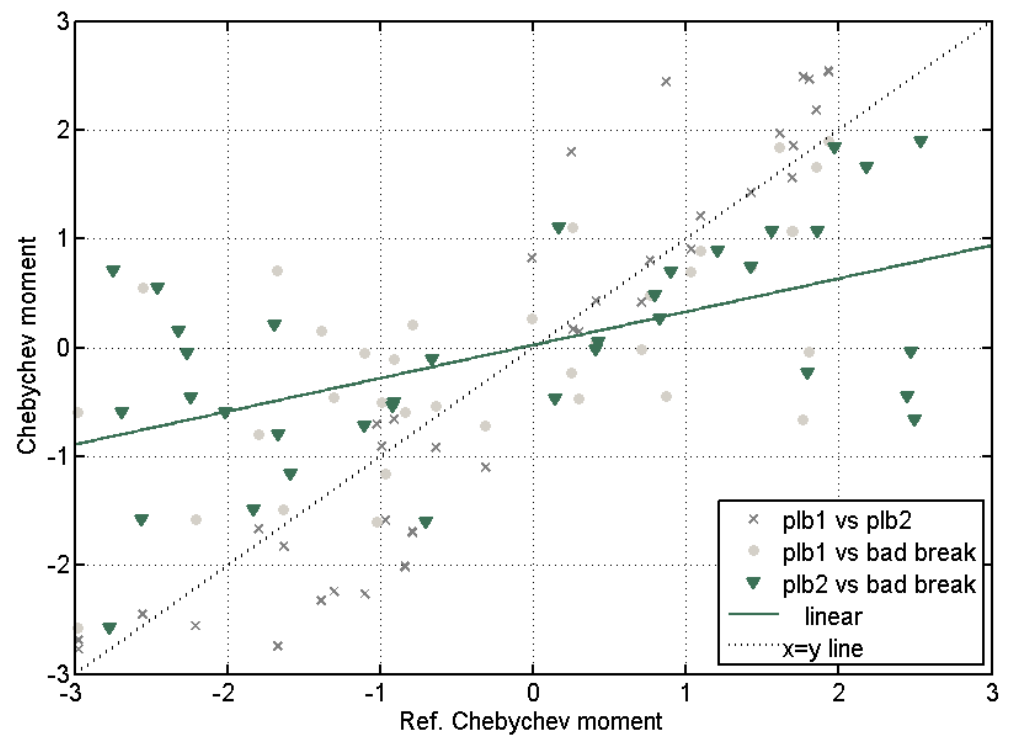

(c)

Figure 6: comparison of different waveforms Chebyshev moments: similar (a) and dissimilar (b and c)

\subsection{Acousto-ultrasonic dataset}

Waveforms amongst the same set proved to have a high degree of similarity, while small but measurable differences were found across sets. Figure 7 shows a baseline waveform superimposed to a waveform received after the $3^{\text {rd }}$ impact.

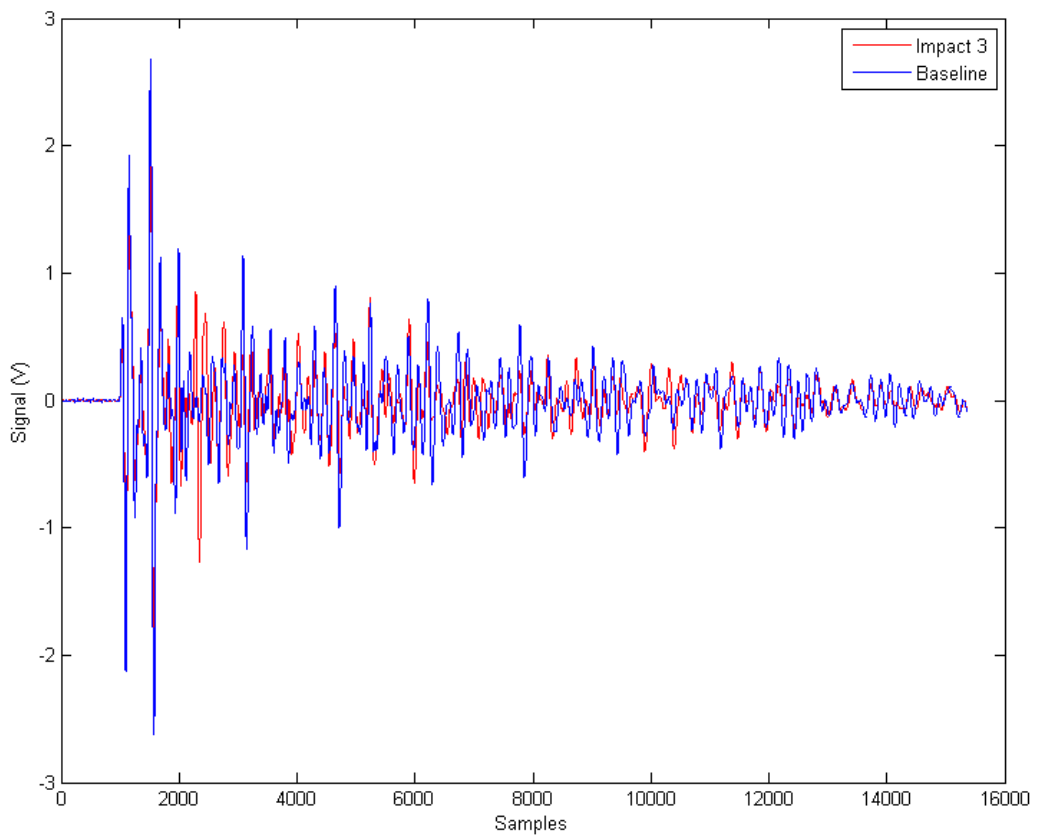

Figure 7: comparison between a baseline and a impact waveform 
The CDs were calculated from a baseline waveform (no damage) and compared with a waveform from the first, second and third impact (increasing impact energy and increasing damage extension). A sample comparison is reported in Figure 8. The correlation coefficients values as a function of the impact order is shown in Figure 9.

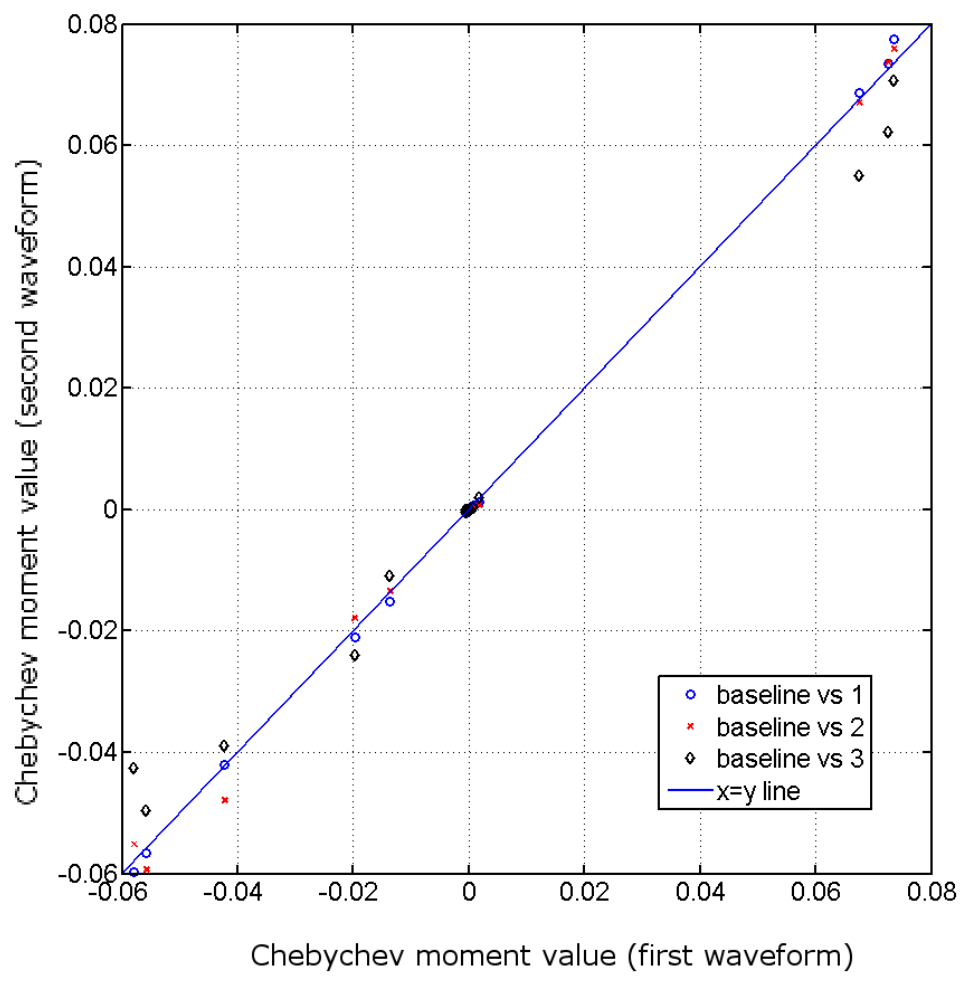

Figure 8: CDs comparison between a baseline waveform and a post-impact waveform 


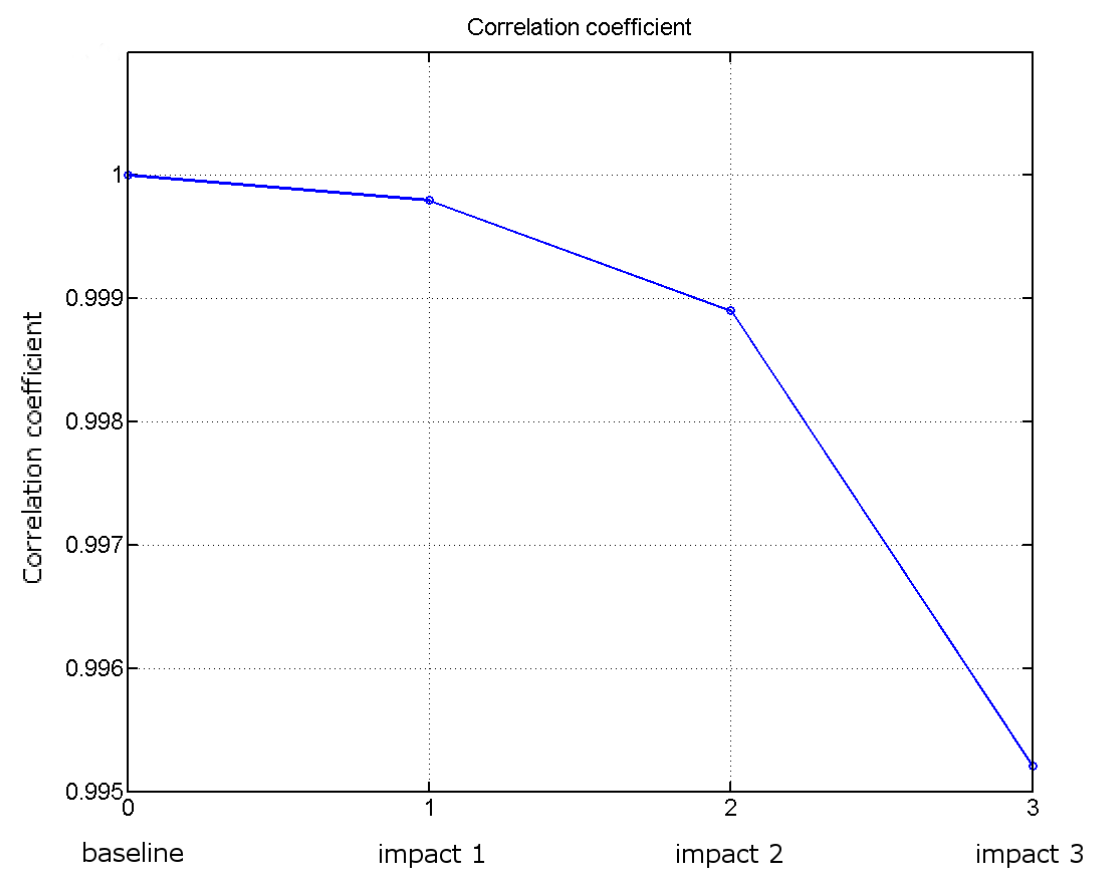

Figure 9: Correlation coefficient of the CDs at various impact levels

The variation of CDs throughout the test was also investigated. In Figure 10 all 36 normalized CDs, for the 100 received waveforms and for test stage going from 1 (baseline) to 4 ( $3^{\text {rd }}$ impact) are shown. 

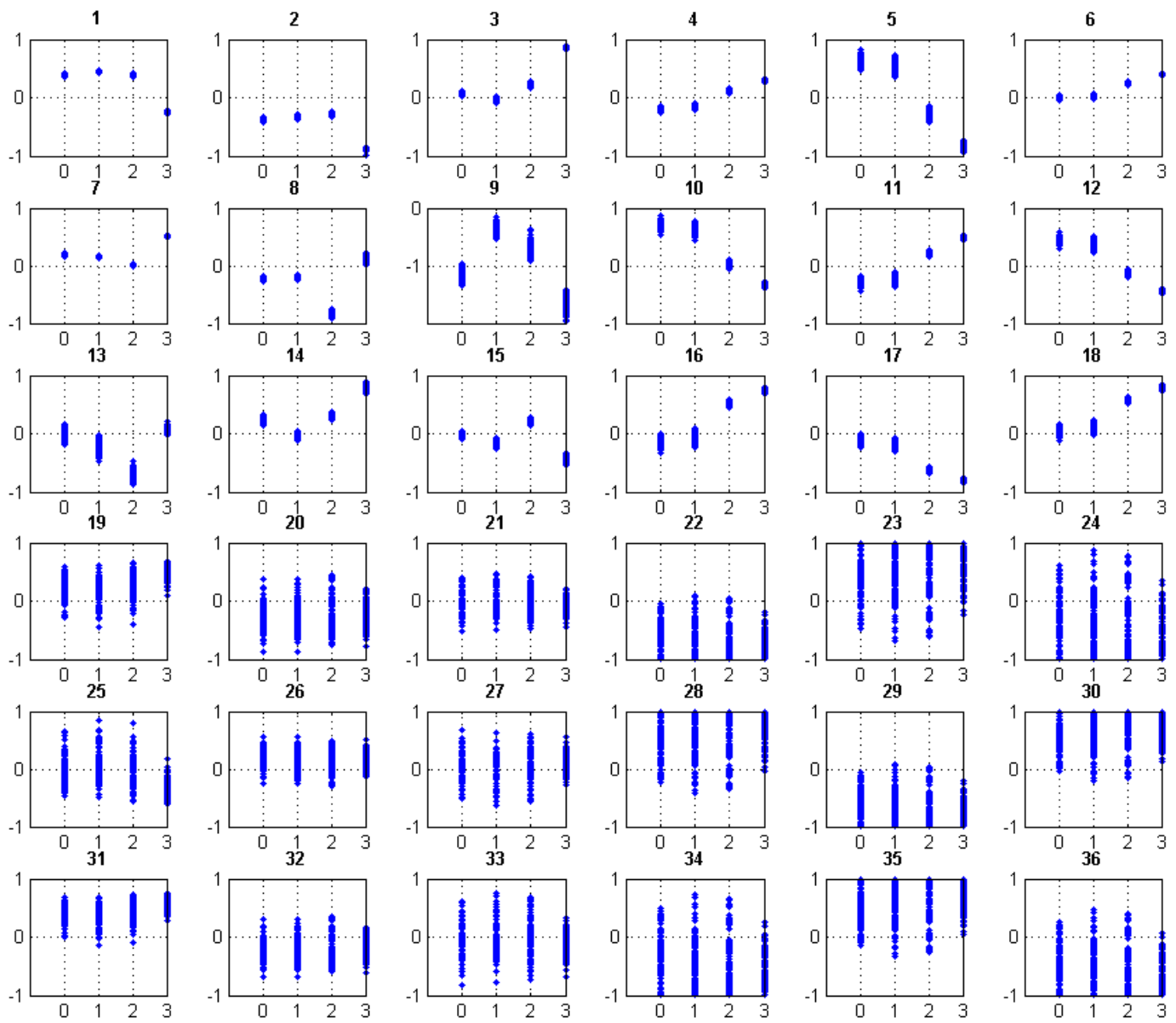

Figure 10: CDs, normalized, for all the 100 waveforms considered in the test

\section{Discussion}

The Acoustic Emission test showed that similar sources (i.e. the good pencil lead break signals) are identified with a high correlation coefficient of their respective CDs, in this case higher than $95 \%$. A bad signal, represented by a double pencil lead break (which is considered invalid during calibration), has significantly lower correlation coefficients of CDs when compared to two different good calibration signals; in this case the values were around $85 \%$. It has to be remarked that a visual inspection of the waveforms doesn't allow an immediate identification of the bad break signal.

In the Acousto-Ultrasonics test, the correlation coefficient of the CDs resemble strongly the cross-correlation decay observed in (Pearson et al. 2011); the same information is, in this case, carried by just 36 parameters instead of having to compare the entire waveforms (consisting of more than 4000 data points each).

Upon inspection of the CDs of the entire dataset, some coefficients are observed to vary significantly at different impact levels, while some appear change randomly. This is mainly because not all wavelet decomposition levels (which are related to frequency bands in the signal) are expected to be influenced by the presence of damage; also, the detail level of 36 in this study appears to be over representing the signals: a lower number of CDs may be enough, depending on the application. 
The data reduction achievable with minimum information loss would be a great benefit for wireless SHM systems, where the power consumption for data transmission might be significantly reduced. The power required for on-board signal processing increases; however, Field Programmable Gate Array (FPGA) processors or dedicated Integrated Circuits (ICs) may be more power efficient than a much longer data transmission over wireless protocols.

\section{Conclusions}

A method for comparing transient waveforms based on the Discrete Wavelet Transform and on the computation of Chebyshev moments has been developed. The method was successful in identifying the difference between regular and failed Acoustic Emission calibration tests, and in comparing waveforms in an Acousto-Ultrasonics setup.

A relationship between the correlation coefficient of the CDs reference pencil lead break versus another good calibration or a bad calibration is apparent from the data shown in this work.

In the Acousto-Ultrasonics test, a relationship between the CDs of a baseline signal (undamaged sample) and subsequent impacts is clear.

The representation of waveforms with a small set of descriptors, in this case the Chebyshev Descriptors, allows a more compact representation of waveforms, and allows comparisons reducing the influence of small time shifts or different triggering of the acquisition. The use of these parameters, instead of the traditional parameters set, will be an advance for remote SHM systems, where data compression is key. Further studies will investigate the effects of various waveform disturbances on the CDs, to verify their robustness to different operating conditions.

\section{Notes}

[1] Alternative graphies found in the literature include: Tchebichef, Tchebycheff, Chebychev, Chebychef.

\section{References}

Bateman, H. et al., 1955. Higher transcendental functions, McGraw-Hill New York.

Crivelli, D., Guagliano, M. \& Monici, A., 2014. Development of an artificial neural network processing technique for the analysis of damage evolution in pultruded composites with acoustic emission. Composites part B, 56, pp.948-959. Available at: http://www.sciencedirect.com/science/article/pii/S1359836813005180.

Daubechies, I., 1988. Orthonormal bases of compactly supported wavelets. Communications on Pure and Applied Mathematics, 41(7), pp.909-996. Available at: http://doi.wiley.com/10.1002/cpa.3160410705 [Accessed March 26, 2014]. 
Downs, K., Hamstad, M. \& O'Gallagher, A., 2003. Wavelet Transform Signal Processing to Distinguish Different Acoustic Emission Sources. Journal of Acoustic Emission, 21, pp.52-69. Available at: http://www.ndt.net/article/jae/papers/21-052.pdf.

Eaton, M.J., 2009. Acoustic Emission Monitoring of Buckling and Failure in Carbon Fibre Composite Structures. Cardiff University, school of Engineering.

Eaton, M.J. et al., 2011. Principal Component Analysis of Acoustic Emission Signals From Landing Gear Components: An Aid to Fatigue Fracture Detection. Strain, 47, pp.e588-e594. Available at: http://doi.wiley.com/10.1111/j.1475-1305.2009.00661.x [Accessed March 18, 2014].

Finleyson, R.D., 2003. Acoustic Emission Testing. In Handbook of Nondestructive Evaluation. McGraw-Hill Companies.

HSU, N.N., 1976. A mechanical AE simulator for system calibration and waveform analysis. In 16th meeting of the U.S. Acoustic Emission Working Group. Williamsburg, Va, United States.

Kapoor, H. et al., 2009. Prospective applications for SHM on Commercial Aircraft. In Proceedings of the 7th International Workshop on Structural Health Monitoring. DEStech Publications, pp. 223-230.

Mukundan, R., Ong, S.H. \& Lee, P.A., 2001. Image analysis by Tchebichef moments. IEEE transactions on image processing : a publication of the IEEE Signal Processing Society, 10(9), pp.1357-64. Available at: http://www.ncbi.nlm.nih.gov/pubmed/18255550 [Accessed February 20, 2014].

Pearson, M.R. et al., 2011. Impact Damage Detection and Assessment in Composite Panels using Macro Fibre Composites Transducers. Journal of Physics: Conference Series, 305, p.012049. Available at: http://stacks.iop.org/1742-6596/305/i=1/a=012049?key=crossref.a818e47ab6a7ac9f941f4e5116045e69 [Accessed March 18, 2014].

Schulz, M.J. et al., 2000. Active fiber composites for structural health monitoring. In C. S. Lynch, ed. Proc. SPIE 3992, Smart Structures and Materials. pp. 13-24. Available at: http://proceedings.spiedigitallibrary.org/proceeding.aspx?articleid=925219 [Accessed March 18, 2014].

Sebastian, C.M., Patterson, E.A. \& Ostberg, D., 2011. Comparison of Numerical and Experimental Strain Measurements of a Composite Panel Using Image Decomposition. Applied Mechanics and Materials, 70, pp.63-68. Available at: http://www.scientific.net/AMM.70.63 [Accessed March 18, 2014].

Stone, D.E.W. \& Dingwall, P.F., 1977. Acoustic emission parameters and their interpretation. NDT International, 10(2), pp.51-62. Available at: http://linkinghub.elsevier.com/retrieve/pii/0308912677900797 [Accessed March 18, 2014].

Yap, P.T. \& Raveendran, P., 2004. Image focus measure based on Chebyshev moments. IEE Proceedings Vision, Image, and Signal Processing, 151(2), p.128. Available at: http://digitallibrary.theiet.org/content/journals/10.1049/ip-vis_20040395 [Accessed March 18, 2014]. 